\title{
Learning-dependent neuronal responses recorded from limbic system brain structures during classical conditioning
}

\author{
THEODORE W. BERGER \\ University of Pittsburgh, Pittsburgh, Pennsylvania 15260 \\ and \\ GREGORY A. CLARK and RICHARD F. THOMPSON \\ University of California, Irvine, California 92717
}

\begin{abstract}
Multiple unit recordings were made from hippocampus and various other limbic system structures during classical conditioning of the rabbit nictitating membrane response. Hippocampal cells increase their firing rate substantially during the very early phases of conditioning-long before behavioral conditioning. A variety of evidence indicates that the hippocampal increase is learning-dependent, suggesting that hippocampal neurons are particularly sensitive to temporal parameters of stimuli used in conditioning. Single unit recordings of physiologically identified hippocampal cells indicate that pyramidal neurons are the cell type exhibiting the increase pattern of activation, while other putative cell types respond in different manners. Recordings from other limbic system brain areas during conditioning show a contrast between activity patterns of the major hippocampal afferents, important similarities between hippocampal and entorhinal cortical within-trial unit patterns of cell activation, and a selective projection of heightened hippocampal discharges along precommissural rather than postcommissurral fornix pathways.
\end{abstract}

In recent years, considerable experimental effort has focused on the stimulus and behavioral conditions that preferentially activate hippocampal neurons (Berger \& Thompson, 1978b; Best \& Best, 1976; O'Keefe, 1976; Olton, Branch, \& Best, 1978; Ranck, 1973; Vinogradova, 1975; Winson \& Abzug, 1978). Our studies of unit activity during classical conditioning of the nictitating membrane (NM) response have concentrated on neuronal plasticity occurring in hippocampal and other limbic structures that is induced by traditional behavioral training paradigms (Gormezano, 1972). In seeming contrast to workers' reporting hippocampal activation by spatial characteristics of an animal's environment (see O'Keefe \& Nadel, 1978), our results indicate that hippocampal neurons are responsive to stimuli ordered in time-

Supported by research grants from the Alfred P. Sloan Foundation (to T.W.B.) and from NIMH (MH26530), NIH (NSI12268), NSF (BNS75-00453), and the McKnight Foundation (to R.F.T.). This work was brought to completion while the last author (R.F.T.) was a fellow of the Center for Advanced Study in the Behavioral Sciences, Stanford, California, with the support of Center funds and funds from NIMH (ST32MH14581-03) and NSF (BNS76-22943 A02). We thank Carol Treanor and Lynn Gale of the Center staff for helpful advice and assistance, as well as Dolores Shirk for manuscript preparation. particularly during learning situations in which a temporal contiguity or contingency exists between the temporally ordered events (e.g., CS-UCS pairing in classical conditioning). Specifically, our studies of hippocampal unit activity during classical conditioning of the NM response have shown that cellular firing increases above spontaneous rates within the very initial stages of paired CS-UCS training, continues to grow to highly significant levels with subsequent conditioning trials, and does not increase when animals are given unpaired control training (Berger, Alger, \& Thompson, 1976; Berger \& Thompson, 1978a). The hippocampal phenomenon is not dependent on the specifics of this particular behavioral paradigm. Rather, it appears to reflect more general properties of learning, in the sense that hippocampal neuronal plasticity is predictably and strongly affected by changes in ISI (Hoehler \& Thompson, 1980), is a long-lasting phenomenon (Kettner \& Thompson, 1978), occurs in more than one species (Patterson, Berger, \& Thompson, 1979), develops to light or tone CS (Coates \& Thompson, 1978), and also occurs when leg flexion (leg-shock UCS) is the required behavioral response (Land, Berger, Patterson, \& Thompson, Note 1). The present paper will synthesize data from a number of completed and ongoing studies which 
address two primary issues concerning the learningdependent hippocampal response that occurs during classical conditioning.

Details concerning the neurophysiological-behavioral methodology and procedures will not be detailed here, as this information is given in previous publications (Berger \& Thompson, 1978a) and in the preceding paper (see Thompson, Berger, Berry, Hoehler, Kettner, \& Weisz, 1980). Briefly stated, multiple unit (chronically implanted electrodes) or single unit (microdrive system with movable electrodes) recordings were taken from various limbic brain regions during acquisition of conditioned NM responding using a tone CS and an airpuff UCS. Nictitating membrane extension was measured by connecting a nylon loop, sewn into the membrane, with the arm of a minipotentiometer mounted on headgear worn by the animal. Membrane movement was thus transduced into a potential change that reflected the amplitude of NM extension throughout the trial duration (i.e., the topography of the behavior). This potential change was frequency modulated and stored on audiotape along with output of unit electrodes. Both brain and behavior data were collected during $250 \mathrm{msec}$ preceding CS onset (pre-CS period), $250 \mathrm{msec}$ between CS and UCS onsets (CS period), and $250 \mathrm{msec}$ following UCS onset (UCS period). The topography of NM movement and the number of simultaneously recorded action potentials were averaged and cumulated, respectively, over eight successively paired CS-UCS occurrences for direct block comparison of unit-behavior activity during conditioning trials. Control animals were given randomized, explicitly unpaired CS and UCS presentations, with unit-NM data collected separately for successive sets of eight CS-alone and eight UCS-alone trials. Analysis of unit data was always computed relative to spontaneous firing levels measured during the nonsignaled, nonstimulus pre-CS period.

\section{Systems Analysis of the Hippocampal Circuit}

The first issue considered here is a "systems analysis" one, related to the projection of hippocampal activity to and from other limbic system structures. Once a learning-dependent neuronal response has been located within the brain, a major question concerns the anatomical "origin" of that response. An increase in cellular activity recorded from a given brain structure might reflect increases in neuronal discharge that are simply "projected" or "relayed" to the recording site from one or more of its major afferents. It is conceivable, then, that the recorded phenomenon of "neuronal plasticity" actually occurs at synaptic sites located in afferent areas. For the hippocampus, possibilities most likely include entorhinal cortex (Hjorth-Simonsen \& Jeune, 1972; Steward, 1976) and medial septal/diagonal band nuclei (Ibata, Desiraju, \& Pappas, 1971; Mosko, Lynch, \& Cotman, 1973; Segal \& Landis, 1974).

A second "systems analysis" issue involves the projection and ultimate target of the hippocampal response along efferent, output pathways. Identification of the limbic and eventual nonlimbic brain regions receiving hippocampal input can give important clues as to the functional significance of hippocampal activity changes recorded during conditioning. The hippocampus proper has two major efferent projection systems, one cortical (to subicular, entorhinal cortices; see Berger, Swanson, Milner, Lynch, \& Thompson, 1980; Swanson \& Cowan, 1977) and one subcortical (to lateral septum; see Meibach \& Siegel, 1977; Swanson \& Cowan, 1977). Projection of the hippocampal response to cortical structures could be considered more consistent with cognitive, "motor programming" brain functions (Moore, 1979; Numan, 1978), whereas projection of hippocampal activity along subcortical pathways is more compatible with notions of telencephalic "modulation" of lower brainstem mechanisms and reflexes (Berger, 1976; Thompson, Berger, Berry, \& Hoehler, 1980). One of the more prominent features of the hippocampal response is the parallel between the pattern of cell firing within conditioning trials and the topography of NM behavior (see Figure 1A upper traces). In light of this correspondence, the latter hypothesis was tested-that is, the projection of hippocampal activity along subcortical pathways to ultimately influence brainstem systems mediating the NM reflex. As a result, initial studies investigated the possible projection of the hippocampal response to subcortical brain areas.

Hippocampal afferents. The former systems analysis issue-the possibility that the neuronal response recorded from hippocampus develops initially in medial septum and/or entorhinal cortex and is projected to hippocampus-will be dealt with first. For this series of studies, multiple unit recordings were made simultaneously from hippocampus and either medial septum or entorhinal cortex (layer II or III) during classical conditioning of the rabbit NM response. Again, the basic objective was to determine if patterns of activity recorded from either or both of the latter two structures were similar to those seen in hippocampus.

Results from medial septal recordings revealed patterns of activity completely different from those simultaneously seen in hippocampus. In the early phases of paired training (see Figure 1A), while hippocampal neurons show an increased pattern of response in the UCS period that parallels NM topography, medial septal cells exhibit onset responses to tone and airpuff. After behavioral conditioning has developed and the NM and hippocampal responses have moved forward 
A.

NM

HIPPOC

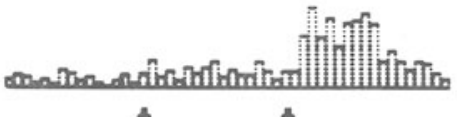

a

M SEPT

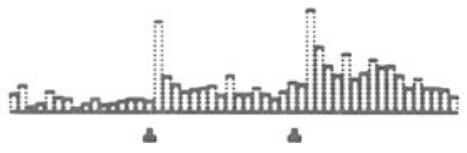

B.

NM

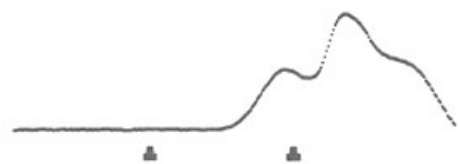

HIPPOC

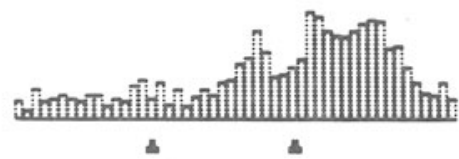

M SEPT

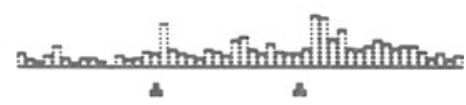

Figure 1. Hippocampal and medial septal unit responses recorded simultaneously from a paired conditioning animal. Upper trace: average nictitating membrane response from one block of eight trials. Middle trace: hippocampal unit poststimulus histogram for one block of eight trials. Lower trace: medial septal unit poststimulus histogram for one block of eight trials. A: First block of paired conditioning trials, Day 1. B: 13th block of paired trials, Day 2. First cursor indicates tone onset; second cursor indicates airpuff onset. Vertical bar in A equals 33 unit counts per 15 -msec time bin. Total trace length equals 750 msec here and in all subsequent histogram figures.

into the CS period (Figure 1B), medial septal activity is still responsive to onsets of the conditioning stimuli. While hippocampal unit activity increases with conditioning, the only change in medial septal activity that develops across trials is a decrease in magnitude of the onset response (Figure 1B). Analysis of unit recordings from animals given unpaired tone-alone and airpuff-alone control training showed that the occurrence of the septal response, its magnitude, and its decrease over trials are not learning-dependent (Figure 2A-D). That is, medial septal units showed sensory-evoked increases in rate that were identical under both paired and unpaired conditions. It is quite apparent, then, that the pattern of neural discharge seen in hippocampus during NM conditioning is not developing first in medial septum. Rather, the medial septal area is responding to stimuli used in the paradigm and could be projecting sensory information to the hippocampus-information that would be critical for the development of neuronal plasticity in any structure.

In contrast to the medial findings, results of simultaneous recordings from hippocampus and entorhinal cortex revealed significant similarities in the activity patterns of the two structures (Clark, Berger, \& Thompson, 1978). Specifically, analyses showed that unit responses in the two brain regions increased above baseline rates during comparable time bins within trials. Early in training, both hippocampus and entorhinal units exhibited increased rates of firing in the UCS period; later in training, units in both areas increased firing rates in the CS and UCS periods.
As a result, the poststimulus trial histograms generated by both recordings paralleled the NM topography. Significant differences between neural responses recorded from the two areas were evident in acrosstrial measures (see Figure 3). That is, while entorhinal activity increased only slightly across training trials, hippocampal activity grew to substantially higher levels. Thus, the specific within-trial pattern of cellular response recorded from hippocampus appears to be projected to it from entorhinal cortex. Hippocampal synapses then "potentiate" or "amplify" that pattern to a much higher magnitude. In a sense, the signal-tonoise ratio of the entorhinal pattern is increased in hippocampus. Whether this neuronal plasticity takes place at the perforant path-dentate synapse or the mossy fiber-CA3 synapse is unknown, as the degree of similarity between dentate and pyramidal cell activity during this paradigm is still uncertain (Berger \& Thompson, 1978a, Note 1).

The results of recordings from entorhinal cortex and hippocampus indicate that the initial systems analysis question posed earlier-that is, whether the unit response recorded from hippocampus develops within hippocampus itself or within entorhinal cortex-may be too simplistic. A given pattern of cell firing that correlates with learning or behavior may not "appear" at one postsynaptic site and not at any presynaptic site (as is true for habituation; e.g., Thompson \& Spencer, 1966). Rather, individual parameters of cellular responding may be contributed by a series of successive synaptic sites. For NM conditioning, the pattern of 
A.

NM

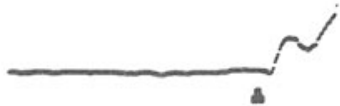

HIPPOC

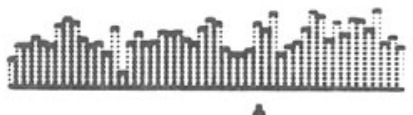

M SEPT

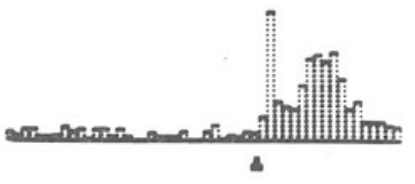

B.

NM

HIPPOC

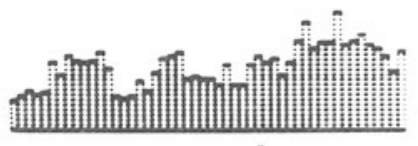

$\Delta$

\section{SEPT}

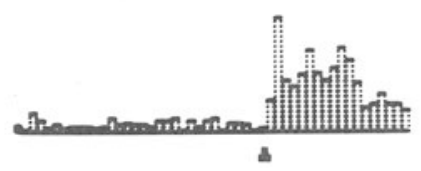

NM

8

C.

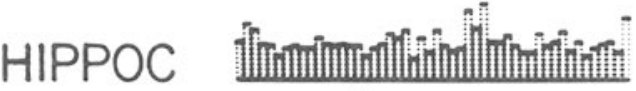

$\triangle$
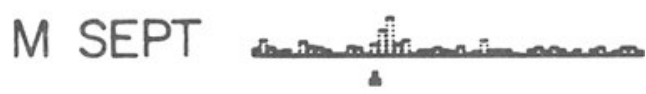

D.

NM

HIPPOC

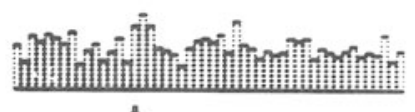

M SEPT

Figure 2. Simultaneously recorded hippocampal and medial septal unit responses from an unpaired control animal. Upper trace: average nictitating membrane response for one block of eight trials. Middle trace: hippocampal unit poststimulus histogram for one block of eight trials. Lower trace: medial septal unit poststimulus histogram for one block of eight trials. A: one block of UCS-alone trials, Day 1. B: one block of UCS-alone trials, Day 2. Cursor indicates airpuff onset. C: one block of CS-alone trials, Day 1. D: one block of CS-alone trials, Day 2. Cursor indicates tone onset. 
UCS PERIOD

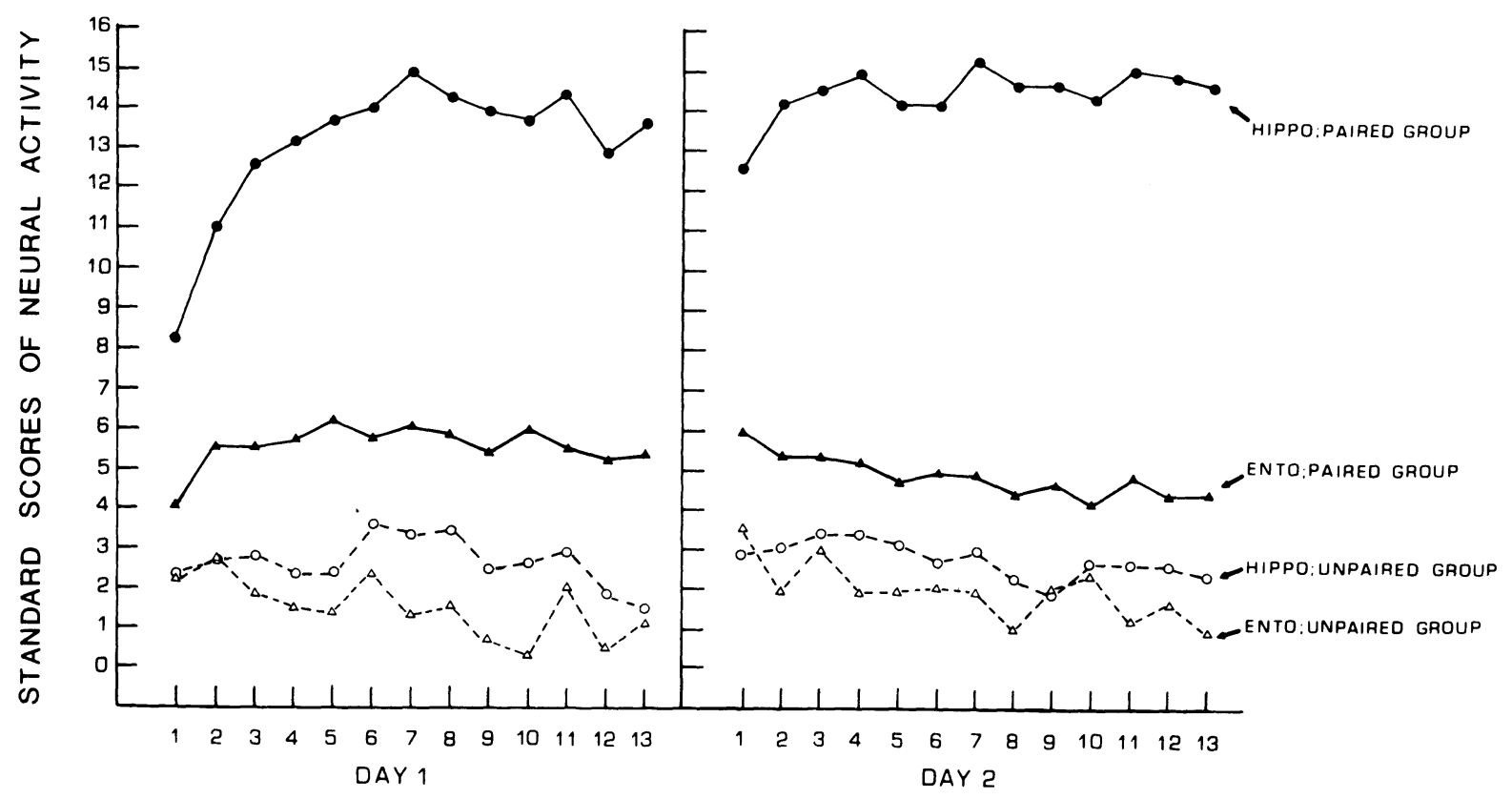

BLOCKS OF TRIALS

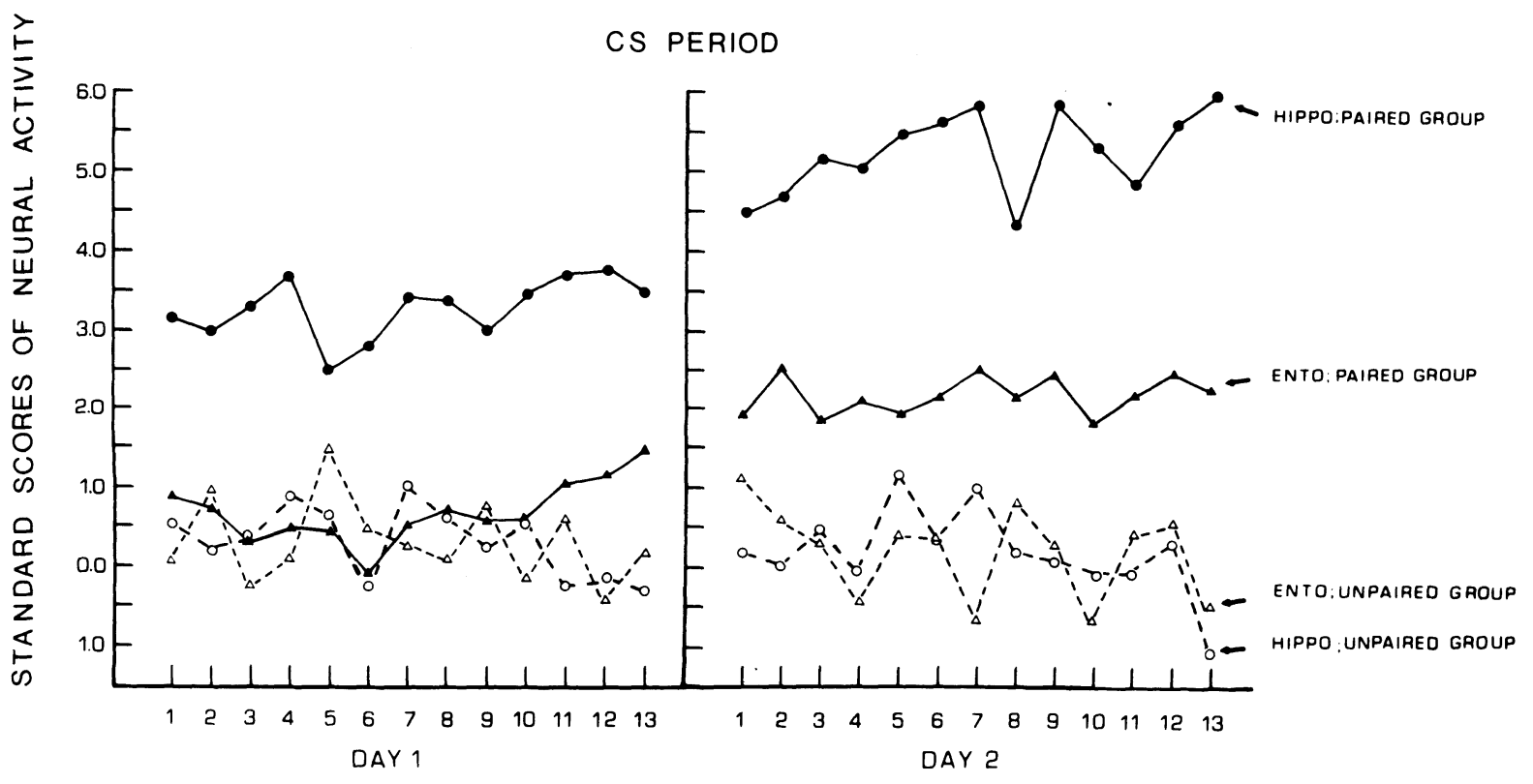

BLOCKS OF TRIALS

Figure 3. Group curves of standard scores of hippocampal and entorhinal neural activity throughout training. Top half: standard scores of unit activity for UCS period. Bottom half: for CS period. Hippocampal activity from paired conditioning group: solid line, filled circles. Entorhinal activity from paired conditioning group: solid lines, filled triangles. Hippocampal activity from unpaired control group: broken lines, open circles. Entorhinal activity from unpaired control group: broken lines, open triangles. 
A.
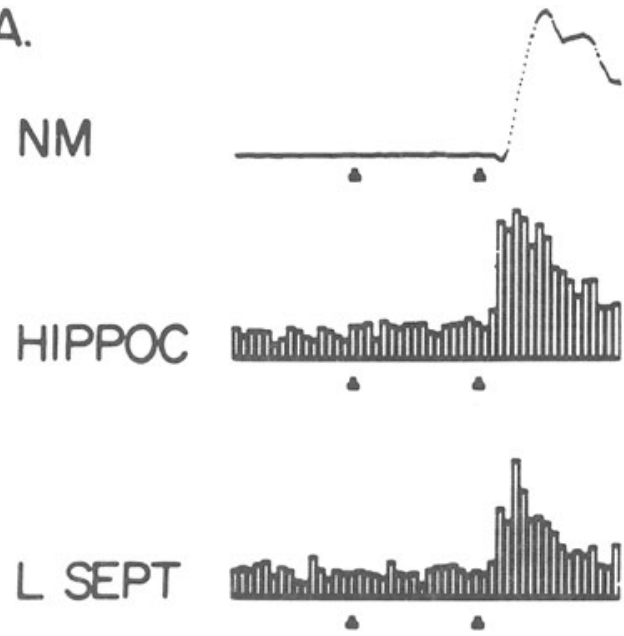

B.

NM
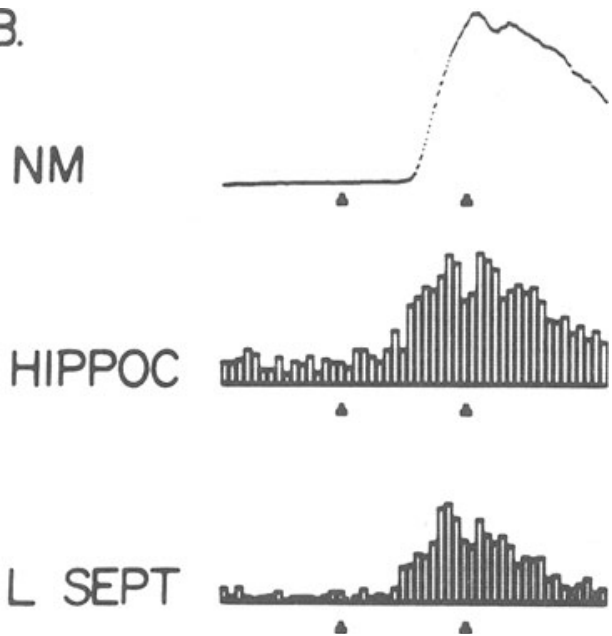

Figure 4. Simultaneously recorded hippocampal and lateral septal unit responses from a paired conditioning animal. Upper trace: average nictitating membrane response for one block of eight trials. Middle trace: hippocampal unit poststimulus histogram (15-msec time bins) for one block of eight trials. Lower trace: lateral septal unit poststimulus histogram (15-msec time bins) for one block of eight trials. A: one block of paired conditioning trials early on Day 1. B: one block of conditioning trials late on Day 1. First cursor indicates tone onset. Second cursor indicates airpuff onset. Vertical bar in A equals 46 unit counts per 15-msec time bin.

cell firing within conditioning trials is contributed by entorhinal cortex, while the substantial increase of that pattern over background firing rates occurs within hippocampus. In fact, further analysis may reveal that only a portion (e.g., the CS or UCS period) of the within-trial pattern is provided by entorhinal cortex, while remaining segments are contributed by entorhinal afferents. These possibilities remain to be investigated.

Hippocampal efferents. The second "systems analysis" issue concerns the projection of the hippocampal response to subcortical limbic system brain regions. The first area investigated-lateral septum-receives monosynaptic input from hippocampal pyramidal cells over the precommissural component of the fornix (DeFrance, Katai, \& Shimono, 1973; Meibach \& Siegel, 1977; Swanson \& Cowan, 1977). Simultaneous multiple unit recordings were made from both structures during the acquisition phase of classical conditioning of the NM response (Berger \& Thompson, $1978 \mathrm{c})$. Results showed a high degree of correspondence between the cellular activity of each anatomical area, both within and across trials (see Figure 4). In both brain regions, increases in unit activity appeared first in the UCS period early in training and in the CS and UCS periods later in conditioning. In both areas, the resulting patterns of activity paralleled the amplitude-time course of the NM response and preceded it in time. Neither hippocampus nor lateral septum increased unit responsiveness under unpaired control conditions (see Figure 5). In total, these results indicate that the hippocampal response is projected at least subcortically along precommissural fornix pathways.

Recordings from anterior thalamic and mammillary nuclei tested the possibility that the hippocampal discharge patterns are projected first to the subicular cortex and then subcortically to postcommissural fornix termination sites. Results of these experiments revealed that none of the above limbic areas showed activity patterns similar to that recorded from hippocampus. Both medial and lateral mammillary nuclei (Figure 6) diffusely increased firing rates only slightly above background frequencies within trial periods and showed only a small decrease in UCS period excitation across trials (Berger \& Thompson, 1978c). An example of results from the anteroventral thalamic nucleus is shown in Figure 7. From the beginning of paired training, a low-amplitude, long-latency (approximately $100 \mathrm{msec}$ from CS onset) unit increase occurred in the CS period and continued throughout the UCS period. Over conditioning trials, the response either stayed constant or decreased. Recordings from the remaining two anterior thalamic nuclei (the anterodorsal and anteromedial) also showed activity patterns that were different from those seen in hippocampus and that did not change significantly with training.

In conclusion, this "systems analysis" of the limbic system during classical conditioning of the NM response has shown that, during acquisition, a specific within-trial pattern of activity can be recorded from hippocampus. The pattern of cell discharge is probably projected from entorhinal cortex to hippocampus, where it is greatly increased in magnitude over back- 


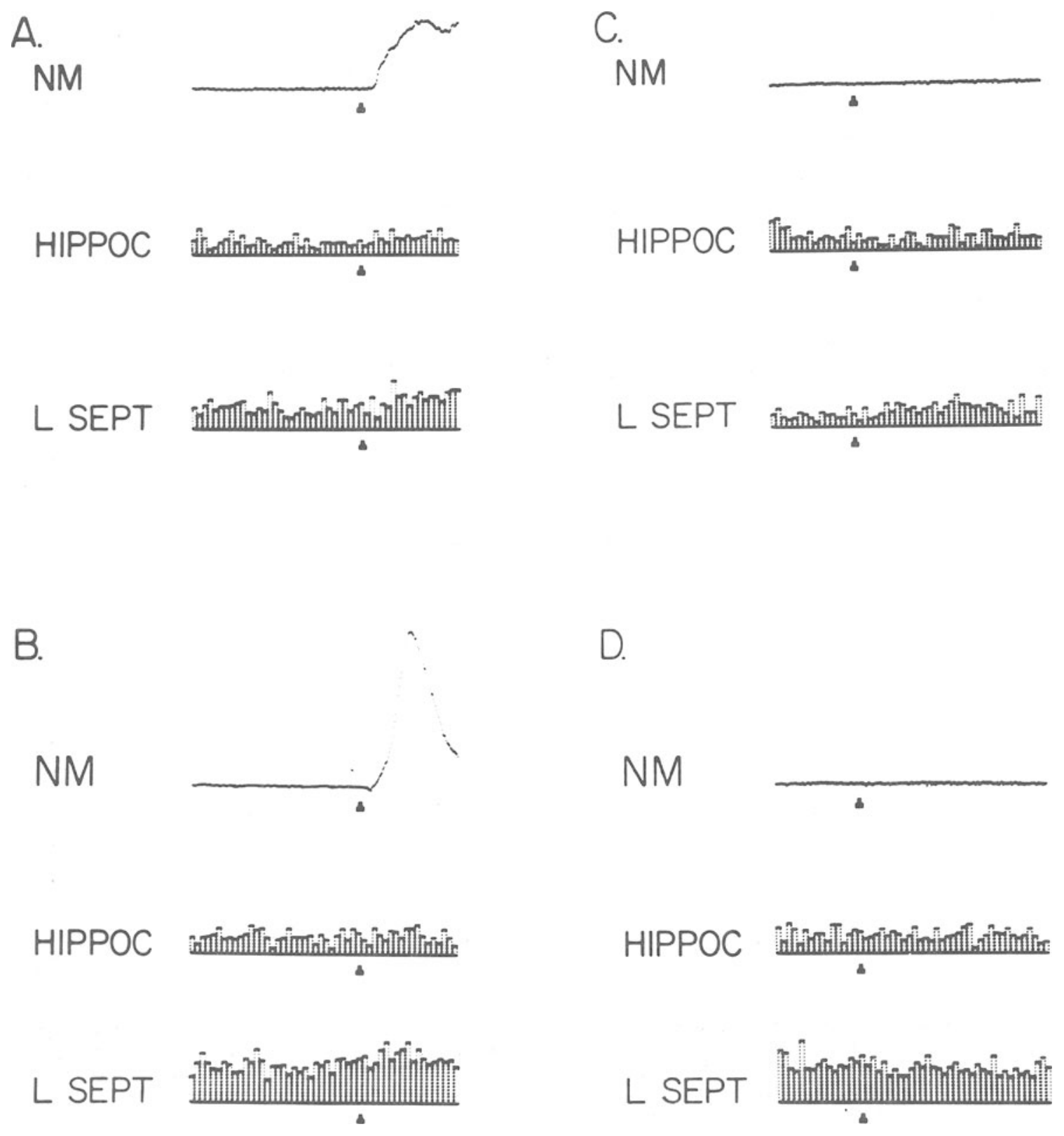

Figure 5. Simultaneously recorded hippocampal and lateral septal unit responses from an unpaired control animal. Upper trace: average nictitating membrane response for one block of eight trials. Middle trace: hippocampal unit poststimulus histogram for one block of eight trials. Lower trace: lateral septal unit poststimulus histogram from one block of eight trials. A: one block of eight UCS-alone trials, Day 1. B: one block of eight UCS-alone trials, Day 2. Cursor indicates airpuff onset. C: one block of eight CS-alone trials, Day 1. D: one block of eight CS-alone trials, Day 2. Cursor indicates tone onset.

ground. From the hippocampus, the unit response is projected selectively over the precommissural aspect of the fornix to at least the level of lateral septum. None of the major subcortical sites receiving postcommissural fornix terminations show activity patterns similar to those recorded from hippocampus. Cell Type Critical to Hippocampal Neuronal Plasticity

The second major issue to be addressed in the present paper relates to the class of cell within the hippocampus that exhibits the specific pattern of discharge seen during NM conditioning. All prior studies were carried out with multiple unit electrodes, which record from small groups of neurons at one time. It is conceivable, in fact likely, that certain cell types within the hippocampus respond with spike distributions similar to those recorded with multiple unit elec- 


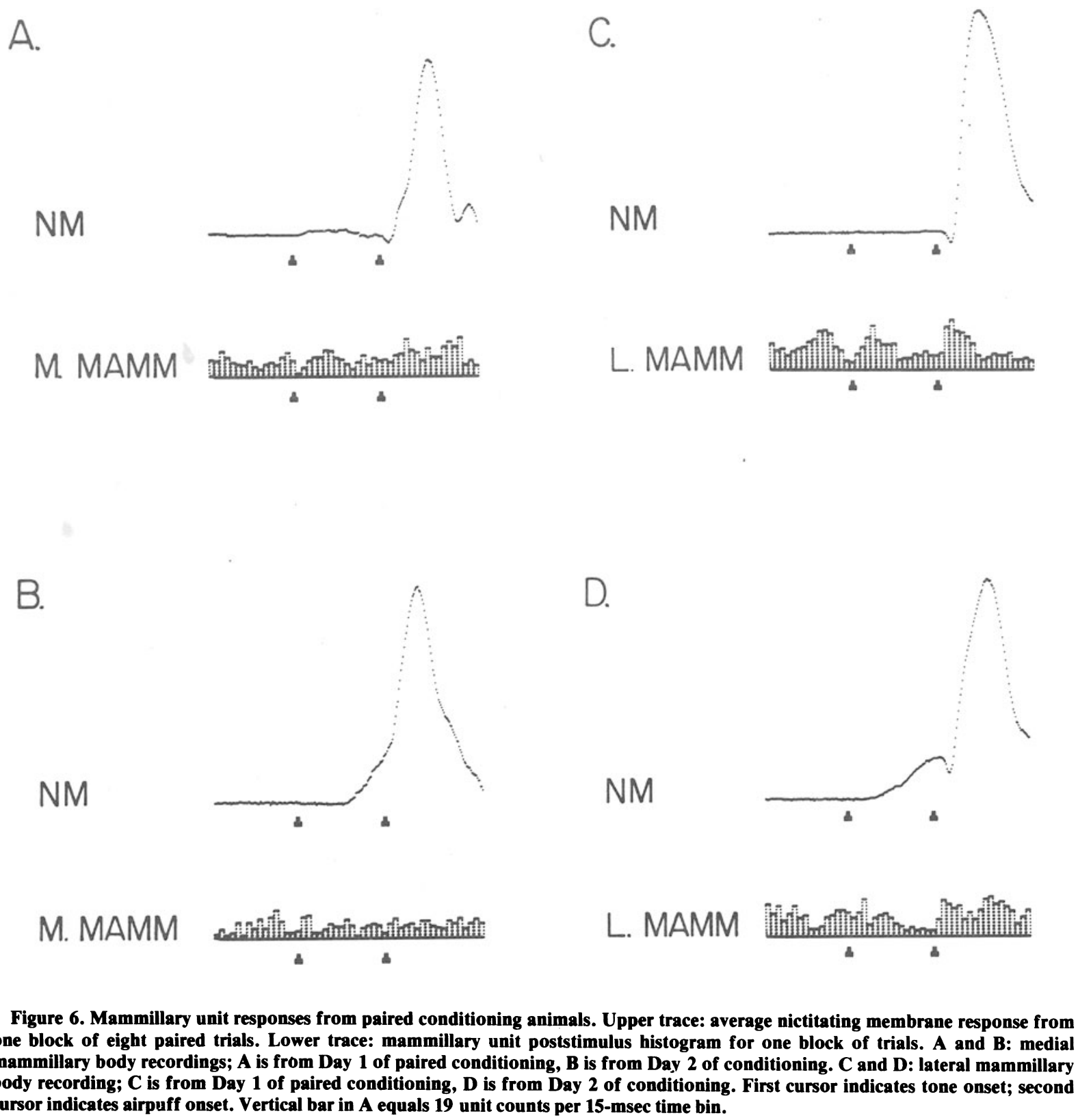

trodes, while other cell types do not. While a number of disparate classes of hippocampal cells have been identified anatomically (Amaral, 1978; Cajal, 1911), only pyramidal (e.g., Kandel, Spencer, \& Brinley, 1961) and granule (e.g., Andersen, Holmqvist, \& Voorhoeve, 1966) cells can be identified directly through physiological methods. Pyramidal cells are the only class of neurons that send axons out of the fornix. Thus, pyramidal cells can be identified conclusively by their antidromic activation (nonsynaptic activation resulting from soma invasion of a spike initiated in the axon) following fornix stimulation. The initial question proposed in this analysis was whether pyramidal cell firing was the basis for the multiple unit activity pattern recorded during NM conditioning trials.
The experimental strategy was to chronically implant bipolar stimulating electrodes into the rabbit fornix, as well as mounting a microdrive system onto the skull. Prior to each conditioning session (animals recovered from surgery for at least 1 week), a single unit electrode (insulated, etched stainless steel; $2-5-\mu$ diam tip, $500 \mathrm{k} \Omega$ to $1 \mathrm{M} \Omega$ impedance) was lowered into the hippocampus and a spontaneously active cell located. The cell was then classified according to its mode of activation following fornix stimulation. Three classifications according to activation were possible. If a cell were antidromically driven, it was classified as a pyramidal neuron. The criteria for antidromic invasion were that the activation latency be low ( $3 \mathrm{msec}$ or less), that the latency display a low variability as a function of stimulus intensity once threshold was reached, 
A.

NM

$\mathrm{CA} 1$

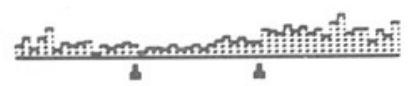

AVTh

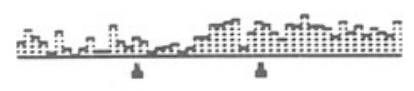

B.

NM

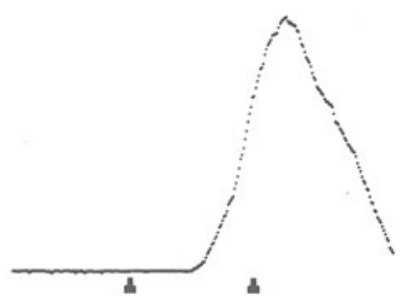

$\mathrm{CA} 1$

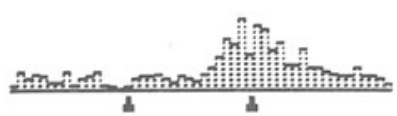

AVTh

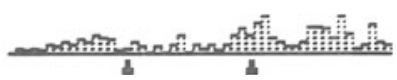

Figure 7. Simultaneously recorded hippocampal and anteroventral thalamic unit responses from a paired conditioning animal. Upper trace: average nictitating membrane response from one block of eight trials. Middle trace: hippocampal unit poststimulus histogram for one block of eight trials. Lower irace: anteroventral thalamic unit poststimulus histogram for one block of eight trials. A: one block of paired conditioning trials early on Day 1. B: one block of conditioning trials late on Day 2. First cursor indicates tone onset. Second cursor indicates airpuff onset.

and that the driven action potential be capable of following high-frequency stimulation $(100 \mathrm{~Hz})$. Only a small proportion of cells were tested for the last criterion, as such a procedure could easily lead to potentiation and seizure effects through activation of afferents to hippocampus. Of those tested, however, cells meeting the first two criteria were also capable of following high-frequency stimulation.

Because afferents to hippocampus also project via the fornix (e.g., from medial septum), certain cells might also be activated orthodromically (i.e., synaptically). Units were classified as orthodromically driven if latencies to activation exceeded $3 \mathrm{msec}$ (most were $4-5 \mathrm{msec}$ ), showed high variability as a function of stimulus intensity, and failed to follow highfrequency stimulation. It should be noted that, while orthodromically activated cells cannot be identified conclusively as any particular cell type, at least a certain percentage could be pyramidal cells that are the target of afferent fornix fibers, but have efferent axons coursing outside the range of the stimulating electrodes.

A third class of cells would include those pyramidal units with axons located outside the range of the fornix electrode, cells receiving fornix afferents over fibers that lie beyond the range of stimulation, and, possibly, interneurons that receive no fornix afferents. As a result, this third class of cells would be expected to show a wide variability in unit response during conditioning.

After each cell had been classified according to fornix stimulation, $3 \mathrm{~min}$ of spontaneous action potentials plus hippocampal slow waves recorded from the same electrode were sampled for later analysis. The objective was to use information concerning the general firing characteristics of a neuron (e.g., spontaneous rate, complex vs. simple firing mode, correlation with hippocampal theta rhythm, etc.) to construct a physiological "profile" to correlate with the performance of the cell during conditioning and its mode of activation following fornix shock (Note 2).

Following this procedure, the animal was trained for as long as the cell could be held (at least 27, and at most 135 , conditioning trials). The unit and behavior data were then collected by a computer and averaged for the total number of trials for which the cell was recorded to produce two final measures, an averaged NM response and an average block poststimulus histogram. Each of these measures is represented in computer storage by $2563-\mathrm{msec}$ bins. A correlation ratio was computed on each set of 256 pairs of points (NM and unit histogram) for each cell. If the resulting ratio was both positive and significant $(p<.05)$, the cell was determined to have a pattern of firing that paralleled the NM topography.

The results of this analysis have shown rather clearly that different subpopulations of hippocampal cells (as defined by fornix stimulation) correlate with different within-trial distributions (Berger \& Thompson, 1978b). Specifically, 78\% (39/50) of hippocampal units classified as pyramidal neurons showed significant, positive correlations with the amplitude-time course of the NM response (see Figure 8 ). The remaining cells inhibited compared with baseline rates $(2 \%)$, maintained spontaneous firing levels during the trial $(10 \%)$, showed significant excitation during conditioning trials but not in a manner that correlated with NM topography $(6 \%)$, or exhibited tone-evoked onset responses $(2 \%)$. The majority of cells in this category exhibited spontaneous firing rates 
A

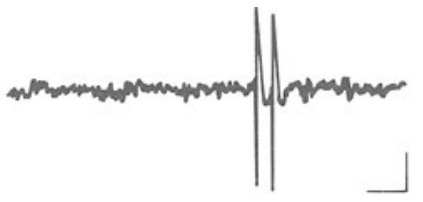

NM
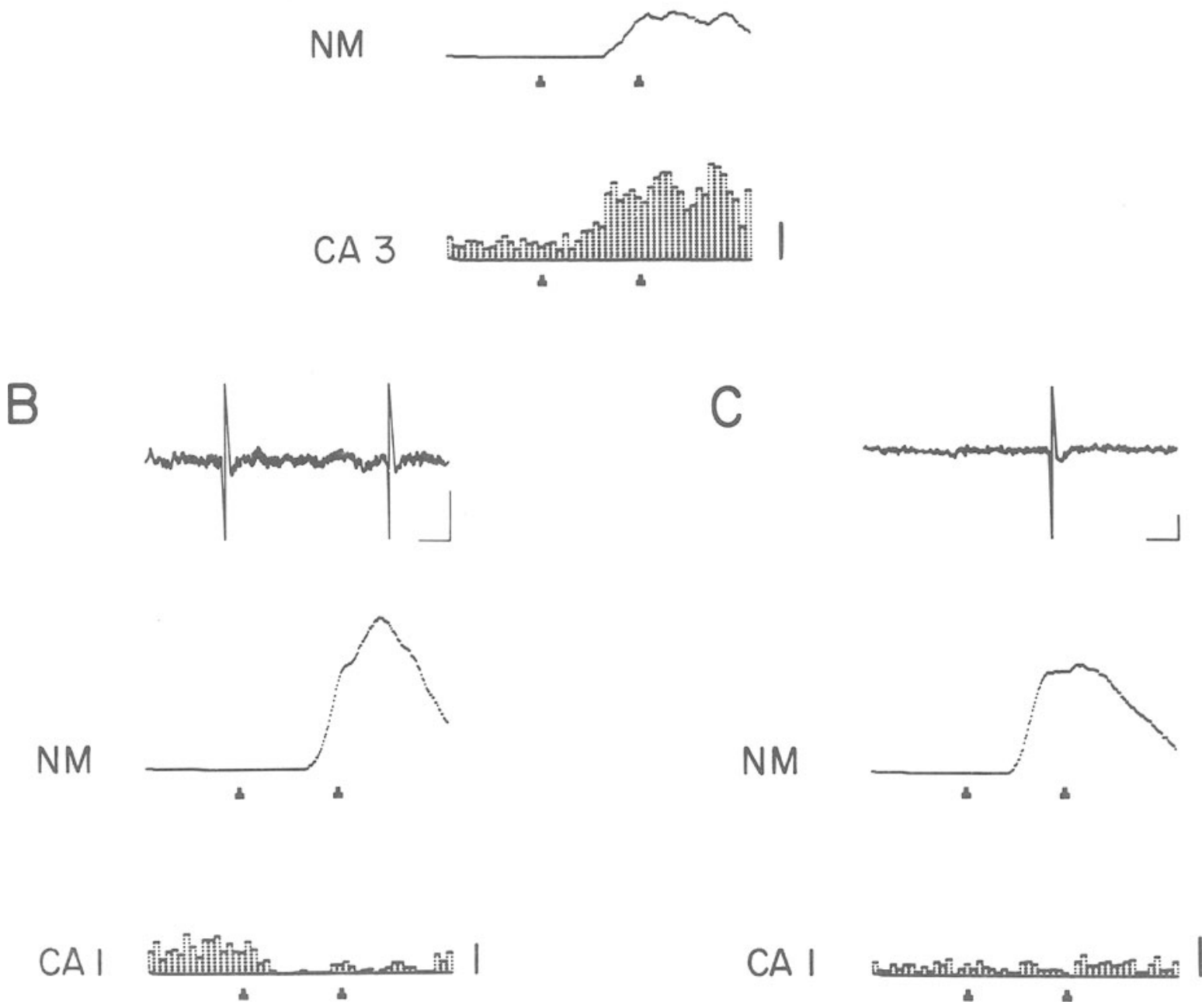

Figure 8. Middle traces show average NM response; bottom traces show poststimulus histograms generated by isolated single hippocampal units recorded during paired conditioning. Top traces show examples of spontaneous activity from the single cells generating the respective histograms. Calibrations for upper raw data traces equal $50 \mu \mathrm{V}$ and $5 \mathrm{msec}$. A: data collected from an antidromically activated unit (pyramidal neuron). Vertical histogram calibration is equivalent to 25 unit counts per 15-msec time bin. B: data collected from an orthodromically activated cell. Calibration equals 21 counts. C: data collected from hippocampal cell not activated by fornix stimulation. Calibration equals 28 counts.

in the 2- to 10-sec range, some cells displayed complex action potentials during pretraining periods, and, in general, the response characteristics of pyramidal cells identified here match those described by other groups (Fox \& Ranck, 1977; Ranck, 1973).

Cells that were orthodromically activated primarily inhibited during trial periods $(11 / 21 ; 52 \%)$. Another $14 \%$ also inhibited during the trial but did not reach statistical significance. Of the remaining cells, $19 \%$ paralleled the NM topography, $5 \%$ diffusely excited during trials, and $10 \%$ showed tone-evoked onset responses. Orthodromically driven cells tended to have higher spontaneous firing rates than pyramidal cells, with the majority of neurons having rates exceeding 10/sec. Again contrasting pyramidal cells, units in the orthodromic category almost always exhibited simple rather than complex spike firing patterns during pretraining periods. It is important to 
note that the differences between orthodromically activated and antidromically driven cells with respect to their spontaneous characteristics and firing patterns during conditioning strongly implicate the orthodromic unit as a nonpyramidal type of hippocampal cell. In fact, the response characteristics of this group generally match those described by Ranck and colleagues (Fox \& Ranck, 1977; Ranck, 1973) as "theta" units, although correlation with theta has not yet been used as a criterion for classification of these cells (Note 2).

Of the recorded neurons that could not be driven by fornix stimulation, $32 \%(11 / 34)$ positively correlated with NM topography and could represent pyramidal cells with axons beyond the stimulation range. Another $18 \%$ inhibited during conditioning trials and could include cells which receive fornix afferents that lie outside the range of stimulation. These are reasonable assumptions, as most cells in the former category had spontaneous response characteristics similar to those described for pyramidal neurons, while those in the latter group had characteristics similar to those described for orthodromically driven cells. A third subgroup which could not be activated orthodromically or antidromically, however, showed response properties that were quite different from those of the previous two groups. Most of the cells in this subgroup had very low spontaneous rates, with levels of $1 / 30-60 \mathrm{sec}$ not uncommon. While pyramidal neurons clearly demonstrated complex spike patterns as a "mode" of firing (i.e., a given pyramidal cell sometimes showed complex spikes and sometimes simple spikes), the majority of low-spontaneous neurons in this third subgroup almost always showed complex spike patterns whenever the cell fired. During conditioning trials, cells in this subgroup typically maintained spontaneous rates or tended toward inhibition. We believe these units to be a separate class of hippocampal neuron that is not capable of being activated (orthodromically or antidromically) by fornix stimulation.

The single unit analysis of physiologically categorized hippocampal cell types clearly implicates pyramidal neurons as the cells that increase their response rate in a specific manner during NM conditioning. Other putative hippocampal cell types respond in different manners. It was pointed out previously that the physiological characteristics of orthodromically activated cells in the present study were similar to those of "theta" cells described by other workers (e.g., Ranck, 1973). Elsewhere, certain evidence has been presented to argue that "theta" cells are hippocampal basket neurons (Fox \& Ranck, 1975, 1977). The present contrast between response patterns of antidromically and orthodromically driven cells during NM conditioning would be consistent with their anatomical classification as pyramidal and basket cells, respectively. Pyramidal collaterals synaptically drive basket cells, which in turn mediate a powerful recurrent inhibition of pyramidal neurons. It is conceivable that strong inhibition of basket cell activity (orthodromically driven cells in the present analysis) is necessary to allow for sustained, repetitive pyramidal cell firing during conditioning trials without activating recurrent inhibitory mechanisms.

\section{Summary}

In total, this analysis has revealed that hippocampal neuronal activity preferentially increases during classical conditioning. Moreover, the increased cellular response takes the form of a specific probability pattern of action potentials within conditioning trials. Evidence indicates that the probability pattern may originate within entorhinal cortex or its afferents and be projected to the hippocampus. Within hippocampus, at either the perforant path-dentate or mossy fiber-CA3 synapse, entorhinal input is "amplified" relative to spontaneous activity rates. This amplification could be mediated either by a greater difference between spontaneous and within-trial frequencies of cell firing in hippocampal neurons vs. entorhinal neurons or by a greater proportion of hippocampal neurons than entorhinal cells increasing firing rate within trials. Single unit analysis indicates that pyramidal neurons are the hippocampal cells responding in a heightened manner during conditioning trials, with a spike distribution that parallels NM topography. This heightened neuronal response is then projected subcortically through hippocampal pyramidal precommissural fornix efferents to lateral septum. None of the subcortical limbic brain regions receiving indirect hippocampal input via the subiculum show unit activation during conditioning similar to that recorded from hippocampal and lateral septum. It is worth pointing out that the subicular cortex (or one of its subdivisions) may respond in a manner similar to hippocampus during conditioning, as this limbic region has yet to be investigated. Emphasis has been placed here on subicular postcommissural fornix projection sites in an effort to integrate current data concerning hippocampal/fornix subcortical projections. These results, in conjunction with others (Berry \& Thompson, 1979; Salafia, Romano, Tynan, \& Host, 1977; also see Salafia \& Allan, 1980), are consistent with a view of the limbic system as becoming selectively activated during learning to modulate, through its heightened efferent output, subcortical brain mechanisms critical to conditioned behavioral responding.

\section{REFERENCE NOTE}

1. Land, T., Berger, T. W., Patterson, M. M., \& Thompson, R. F. Learning-dependent increases in hippocampal unit activity during classical tone-footshock conditioning in the rabbit. Manuscript in preparation. 


\section{REFERENCES}

Amaral, D. G. A golgi study of cell types in the hilar region of the hippocampus in the rat. Journal of Comparative Neurology, 1978, 182, 851-914.

Andersen, P., Holmqvist, B., \& Voorhoeve, P. E. Entorhinal activation of dentate granule cells. Acta Physiologica Scandinavica, 1966, 66, 448-460.

BERGER, T. W. Neuronal plasticity in the limbic system during classical conditioning. Unpublished doctoral dissertation, Harvard University, 1976.

Berger, T. W., Alger, B., \& Thompson, R. F. Neuronal substrate of classical conditioning in the hippocampus. Science, 1976, 192, 483-485.

Berger, T. W., Swanson, G. W., Milner, T. A., Lynch, G. S., \& Thompson, R. F. Reciprocal anatomical connections between hippocampus and subiculum in the rabbit: Evidence for subicular innervation of regio superior. Brain Research, 1980, 183, 265-276.

Berger, T. W., \& Thompson, R. F. Neuronal plasticity in the limbic system during classical conditioning of the rabbit nictitating membrane response. I. The hippocampus. Brain Research, 1978, 145, 323-346. (a)

Berger, T. W., \& Thompson, R. F. Identification of pyramidal cells as the critical elements in hippocampal neuronal plasticity during learning. Proceedings of the National Academy of Sciences (Washington), 1978, 75, 1572-1576. (b)

Berger, T. W., \& Thompson, R. F. Neuronal plasticity in the limbic system during classical conditioning of the rabbit nictitating membrane response. II. Septum and mammillary bodies. Brain Research, 1978, 156, 293-314. (c)

Berry, S. D., \& Thompson, R. F. Medial septal lesions retard classical conditioning of the nictitating membrane response in rabbits. Science, 1979, 205, 209-211.

BEst, M. R., \& BEST, P. J. The effects of latent inhibition on hippocampal unit activity in the rat. Experimental Neurology, 1976, 51, 546-573.

CAJAL, R. Y. Histologie du système nerveux de l'homme et des vertebres. Paris: Maloine, 1911.

Clark, G. A., Berger, T. W., \& Thompson, R. F. Role of entorhinal cortex during classical conditioning: Evidence for entorhinal-dentate facilitation. Neuroscience Abstracts, 1978, 4, 217.

Coates, S. R., \& Thompson, R. F. Comparing neuronal plasticity in the hippocampus during classical conditioning of the rabbit nictitating membrane response to light and tone. Neuroscience Abstracts, 1978, 4, 256.

DeFrance, J. F., Katai, S. T., \& Shimono, T. Electrophysiological analysis of the hippocampal-septal projections. I. Response and topographical characteristics. Experimental Brain Research, 1973, 17, 463-476.

Fox, S. E., \& RANCK, J. B., JR. Localization and anatomical identification of theta and complex spike cells in dorsal hippocampal formation of rats. Experimental Neurology, 1975, 49, 299-313.

Fox, S. E., \& RANCK, J. B., JR. Hippocampal complex-spike and theta cell activity evoked by stimulation of limbic structures in unrestrained rats. Neuroscience Abstracts, 1977, 3, 198.

Gormezano, I. Investigations of defense and reward conditioning in the rabbit. In A. H. Black \& W. F. Prokasy (Eds.), Classical conditioning II: Current research and theory. New York: Appleton-Century-Crofts, 1972.

Huorth-Simonsen, A. Hippocampal efferents to the ipsilateral entorhinal area: An experimental study in the rat. Journal of Comparative Neurology, 1971, 142, 417-438.

Huorth-Simonsen, A., \& JEUNE, B. Origin and termination of the hippocampal perforant path in the rat studied by silver impregnation. Journal of Comparative Neurology, 1972, 144, 215-232.

Hoenler, F. K., \& Thompson, R. F. Effect of interstimulus
(CS-UCS) interval on hippocampal unit activity during classical conditioning of the nictitating membrane response in rabbit, Oryctolagus cuniculus. Journal of Comparative and Physiological Psychology, 1980, 94, 201-215.

Ibata, Y., Desiraju, T., \& Pappas, G. D. Light and electron microscopic study of the projection of the medial septal nucleus to the hippocampus of the cat. Experimental Neurology, 1971, 33, 103-122.

Kandel, E. R., Spencer, W. A., \& Brinley, F. J. Electrophysiology of hippocampal neurons. I. Sequential invasion and synaptic organization. Journal of Neurophysiology, 1961, 24, 225-242.

Kettner, R. E., \& Thompson, R. F. Neural correlates of a signal detection task in the rabbit. Journal of the Acoustical Society, 1978, 64, S137.

Meibach, R. C., \& Siegel, A. Efferent connections of the hippocampal formation in the rat. Brain Research, 1977, 124, 197-224.

MOoRE, J. W. Brain processes and conditioning. In A. Dickinson \& R. A. Boakes (Eds.), Associative mechanisms in conditioning. Hillsdale, N.J: Erlbaum, 1979.

Mosko, S., Lynch, G., \& Cotman, C. W. The distribution of septal projections to the hippocampus of the rat. Journal of Comparative Neurology, 1973, 152, 163-174.

Numan, R. Cortico-limbic mechanisms and response control: A theoretical review. Physiological Psychology, 1978, 6, 445-470.

O'KEEFE, J. Place units in the hippocampus of the freely moving rat. Experimental Neurology, 1976, 51, 78-109.

O'KeEFE, J., \& NADEL, L. The hippocampus as a cognitive map. Oxford: Clarendon Press, 1978.

Olton, D. S., Branch, M., \& Best, P. J. Spatial correlates of hippocampal unit activity. Experimental Neurology, 1978, 58, 387-409.

Patterson, M. M., Berger, T. W., \& Thompson, R. F. Neuronal plasticity in the cat hippocampus during classical conditioning. Brain Research, 1979, 163, 339-343.

RANCK, J. B., JR. Studies on single neurons in dorsal hippocampal formation and septum in unrestrained rats. I. Behavioral correlates and firing repertoires. Experimental Neurology, 1973, 41, 461-531.

Salafia, W. R., \& Allan, A. M. Conditioning and latent inhibition with electrical stimulation of hippocampus. Physiological Psychology, 1980, 8, 247-253.

Salafia, W. R., Romano, A. G., Tynan, T., \& Host, K. C. Disruption of rabbit (Oryctolagus cuniculus) nictitating membrane conditioning by posttrial electrical stimulation of hippocampus. Physiology \& Behavior, 1977, 18, 207-212.

Segal, M., \& Landis, S. Afferents to the hippocampus of the rat studied with the method of retrograde transport of horseradish peroxidase. Brain Research, 1974, 78, 1-15.

STEWARD, O. Topographic organizations of the projections from the entorhinal area to the hippocampal formation in the rat. Journal of Comparative Neurology, 1976, 167, 295-314.

Swanson, L. W., \& Cow AN, W. M. An autoradiographic study of the organization of efferent connections of the hippocampal formation in the rat. Journal of Comparative Neurology, 1977, 172, 49-84.

Swanson, L. W., Wyss, J. M., \& Cowan, W. M. An autoradiographic study of the organization of intrahippocampal association pathways in the rat. Journal of Comparative Neurology, 1978, 181, 681-716.

Thompson, R. F., Berger, T. W., Berry, S. D., \& Hoehler, F. K. The search for the engram. II. In D. McFadden (Ed.), Neural mechanisms in behavior: A Texas symposium. New York: Springer-Verlag, 1980 , in press.

Thompson, R. F., Berger, T. W., Berry, S. D., Hoehler, F. K., Kettner, R. E., \& Weisz, D. J. Hippocampal substrate of classical conditioning. Physiological Psychology, 1980, 8, 262-279. 
Thompson, R. F., \& Spencer, W. A. Habituation: A model phenomenon for the study of neuronal substrates of behavior. Psychological Review, 1966, 173, 16-43.

Vinog RAdova, O. S. Functional organization of the limbic system in the process of registration of information: Facts and hypotheses. In R. L. Isaacson \& K. H. Pribram (Eds.), The hippocampus (Vol. 2). New York: Plenum Press, 1975.

Winson, J., \& Abzug, C. Neuronal transmission through hippocampal pathways dependent on behavior. Journal of Neurophysiology, 1978, 41, 716-732.

\section{NOTES}

1. It should be noted that the hippocampus proper has extensive reciprocal connections with entorhinal cortex (Hjorth-Simonsen, 1971; Swanson, Wyss, \& Cowan, 1978). While the entorhinal layers (II and III) recorded from in the study described here have been documented as primarily afferents to hippocampus, the possibility of interactions between entorhinal layers receiving efferents from hippocampus and layers II and III remains unresolved.

2. Analysis of spike correlations with theta rhythm is in progress and will not be presented here. 